\title{
WEARING KNEE SLEEVES DURING BACK SQUATS DOES NOT IMPROVE MASS LIFTED OR AFFECT KNEE BIOMECHANICS
}

original paper

( ) University School of Physical Education in Wroclaw

DOI: https://doi.org/10.5114/hm.2021.100012

\section{HUNTER J. BENNETT ${ }^{1}$, ALEXANDRIA TRYPUC ${ }^{1}$, KEVIN A. VALENZUELA ${ }^{2}$, ZACHARY A. SIEVERT ${ }^{1}$}

${ }^{1}$ Old Dominion University, Norfolk, USA

${ }^{2}$ California State University Long Beach, Long Beach, USA

\begin{abstract}
Purpose. Knee sleeves have become widely popular in the exercise realm, especially for knee support during back squats. Knee sleeves are successful in providing frontal plane knee support during functional tasks, but have not been investigated in back squats. Knee wraps, a somewhat similar elastic material, provide elastic energy that increases weight lifted during back squats. Thus, it is possible the thick neoprene knee sleeves could prove advantageous for back squats. The purpose of this study was to examine the effects of knee sleeves on weight lifted, knee biomechanics, and muscle activations during back squats.
\end{abstract}

Methods. Fifteen resistance-trained men and women performed 1-repetition maximum (1RM) squats to full depth and $80 \%$ 1RM to full and parallel depths during 2 separate randomized sessions: with/without knee sleeves. Three-dimensional motion capture, force platforms, and electromyography recorded knee biomechanics and activations of the rectus femoris, vastus medialis, biceps femoris long head, and gluteus maximus during all squats.

Results. Maximal weight lifted did not improve when using knee sleeves. Frontal plane knee biomechanics did not differ for $1 \mathrm{RM}$ or submaximal squats to either depth between conditions. Knee external rotation moments during descent were larger with sleeves during submaximal squats. Reduced integrated ascent phase gluteus maximus activations occurred during both 1RM and submaximal squats with knee sleeves.

Conclusions. The results of this study show that wearing knee sleeves does not provide additive effects to weight lifted and does not appear to alter frontal plane mechanics during weighted back squats.

Key words: knee sleeves, knee biomechanics, back squats, squat performance

\section{Introduction}

The squat is a widely used exercise in athletics and other exercise regimens. It is a complex exercise that involves all degrees of freedom of the lower body [1-4] and targets several muscles of the lower body [3-5]. The squat has been the subject of many biomechanical evaluations in the areas of kinetics, kinematics, and muscle recruitment and in various stance and load conditions [4]. The primary muscles involved in squats surround the hip and knee joints (e.g. quadriceps, gluteal muscles), which generate $80-90 \%$ of the lower extremity moments [2, 6-8]. In addition to stance and load variations, many training accessories exist for the squat that athletes and recreational exercisers use for enhancing training or preventing injury.

Knee wraps are a weightlifting accessory primarily used in heavy back squats to enhance knee stability, as well as improve performance and/or confidence [9-11]. Knee wraps have previously been found to increase peak power output during back squats through the usage of elastic energy [9]. Storage of elastic energy during the lowering phase can then be released during ascent as kinetic energy, resulting in increased vertical impulses (force ${ }^{*}$ time) and increased 1-repetition maximum (1RM) mass lifted $[9,10]$. Research has also found a muscular response to knee wraps [5]. Comparing vastus lateralis and gluteus maximus activity

Correspondence address: Hunter J. Bennett, Old Dominion University, 1007 Student Recreation Center, Norfolk, VA, USA, e-mail: hjbennet@odu.edu

Received: October 4, 2019

Accepted for publication: July 13, 2020

Citation: Bennett HJ, Trypuc A, Valenzuela KA, Sievert ZA. Wearing knee sleeves during back squats does not improve mass lifted or affect knee biomechanics. Hum Mov. 2021;22(2):32-42; doi: https://doi.org/10.5114/hm.2021.100012. 
during back squats with and without knee wraps, using knee wraps resulted in increased vastus lateralis and decreased gluteus maximus activations when squatting at $90 \% 1 \mathrm{RM}$ [5]. Although beneficial for weightlifting, knee wraps require specific wrapping techniques [10] and could involve significant occlusion of the knee joint [12]. In addition, the significant changes to hip and knee biomechanics when using knee wraps [5, 9], coupled with alterations in vastus lateralis activation [5], could negatively impact on joint stability during weight training programs. Neoprene knee sleeves are another popular knee support device but have not been as widely investigated in weighted exercise.

Knee sleeves are compression-style garments worn around the knee joint. Despite a lack of supportive research, knee sleeves are marketed by manufactures as devices that can provide compression and support to the knee joint for stability, while also aiding performance by increasing weight lifted, repetitions, and training intensity. Although these aims have not been fully addressed in exercise settings, a few research studies have evaluated the efficacy of knee sleeves in other aspects.

From a clinical standpoint, persons with knee osteoarthritis displayed improved joint position sense, pain, stiffness, and function while wearing thin elastic knee sleeves compared with those without sleeves during level walking [13-16]. Using the visual analogue scale, participants also reported feeling less pain while walking with knee sleeves compared with no sleeves [13]. With reference to knee biomechanics, persons with knee osteoarthritis displayed reduced knee adduction angles, first peak external knee adduction moments, and knee adduction impulses when wearing sleeves than with no sleeves during walking [15]. In healthy populations, knee sleeves can provide a small resistive support to unwanted anterior tibial translations [17]. Thus, in low-loading situations, knee sleeves appear to provide enhanced control of both sagittal and frontal plane tibial translations/loading. Should knee sleeves provide support to the knee joint during more dynamic tasks such as weightlifting, this support could manifest as (1) a reduction of abnormal frontal plane loading (moments and powers) and/or (2) greater control of tibial translations. However, as emphasized earlier, research has yet to evaluate the effects of knee sleeves on frontal plane knee biomechanics.

Despite supportive research on knee sleeves in lowloading tasks, support regarding improvements in exercise performance does not exist. Only one previous study has evaluated the effects of knee sleeves during exercise [18]. In this study, knee kinematics and predicted muscle forces during submaximal squats were compared between wearing knee sleeves, competitive knee wraps, training knee wraps, and nothing covering the knees [18]. Knee sleeves provided no apparent advantage (e.g. bar speed) compared with natural lifting and presented no meaningful differences compared with either knee wrap [18]. While the previous study provides evidence to refute claims for the performance aspect of knee sleeves, future work is still required to fully address these claims. For instance, in the previous study, participants lifted $70 \%$ of a self-reported 1RM and performed squats to parallel [18]. Squat depth affects knee biomechanics and muscle activation patterns $[1,2,19,20]$. Both squat depth and a heavier loading scheme could influence the elastic rebound provided by knee sleeves. In this respect, any discernible advantage of knee sleeves would be present as greater knee moments (beyond that of natural conditions) and angular velocities (i.e. joint powers). However, knee moments and powers have not been analyzed. In addition to these measures, muscle activation and knee biomechanics across the supportive planes (i.e. frontal and transverse) have yet to be evaluated. Considering the frequency of knee sleeves in major competitions (e.g. CrossFit Games) and in the general population for squats and similar exercises, research is warranted to evaluate the efficacy of knee sleeves in both maximal and submaximal weightlifting.

Therefore, the purpose of this research was to assess the effects of wearing neoprene knee sleeves on maximal mass lifted, lower body biomechanics, and muscle activations during back squats. As the gluteus maximus is extensively involved in the back squat [2, $19,20]$ and previous reports have found knee wraps can influence its activations [5, 9], this analysis will include the gluteus maximus musculature in addition to surrounding knee musculature. On the basis of the support and improved functions knee wraps and thinner knee sleeves provide, we hypothesized that: (1) maximal mass lifted would increase with knee sleeves, (2) frontal and transverse plane knee joint loading would reduce with knee sleeves, and (3) the support provided by the knee sleeve would reduce knee extensor activations.

\section{Material and methods}

\section{Participants}

Fifteen healthy, resistance-trained individuals were recruited from the local Hampton Roads fitness com- 
H.J. Bennett, A. Trypuc, K.A. Valenzuela, Z.A. Sievert, Knee sleeves do not affect knee mechanics

munity, including the university campus (mass: $82.43 \pm$ $14.37 \mathrm{~kg}$, height: $1.73 \pm 0.12 \mathrm{~m}$, leg length [anterior superior iliac spine to medial malleolus]: $0.89 \pm 0.06$ $\mathrm{m})$. The inclusion criteria involved: being healthy with no history of knee injuries, age $18-55$ years, resistance training at least 3 times a week, at least 2 of the training days including lower body exercises, and at least 1-year experience in back squatting at maximal or near-maximal loads. The exclusion criteria were the following: any major lower extremity musculoskeletal injury in the past 3 months, knee pain in the past 6 months during activities of daily living, a diagnosis of lower extremity joint arthritis, a body mass index greater than $35 \mathrm{~kg} \cdot \mathrm{m}^{-2}$. All participants were informed of the study procedures, risks, and benefits.

\section{Instrumentation}

A 10-camera motion capture system $(200 \mathrm{~Hz}$, Vicon Motion Analysis Inc., Oxford, UK) and 2 force platforms (2000 Hz, Bertec FP-4060, Bertec Inc., Columbus, USA) were used to collect 3-dimensional kinematics and ground reaction forces (GRFs) during 2 testing sessions (further information below). The participants wore a pair of standardized laboratory shoes (Nike Air Max Glide), tight-fitting spandex shorts, and no shirt (males)/ sports top (females). Retroreflective anatomical markers were placed bilaterally on the acromion processes, iliac crests, anterior superior iliac spines, posterior superior iliac spines, greater trochanters, femoral epicondyles, medial and lateral femoral condyles, medial and lateral malleoli, $1^{\text {st }}$ and $5^{\text {th }}$ metatarsal heads, and $2^{\text {nd }}$ toes. Clusters of 4 tracking markers were attached to the posterior trunk, pelvis, thighs, shanks, and shoe heels. The anatomical and tracking markers were used to create a biomechanical model consisting of 8 segments (trunk, pelvis, thighs, shanks, and feet) with 6 degrees of freedom each [21]. Body weight was determined with the force platforms.

A 16-channel electromyography (EMG) system (2000 Hz, Delsys Trigno, Delsys Inc.) collected muscle activity data of 5 muscles on the right leg: vastus medialis, rectus femoris, gluteus maximus, gluteus medius, and biceps femoris. Electrode placements followed the Surface ElectroMyoGraphy for the NonInvasive Assessment of Muscles (SENIAM) guidelines, including shaving, abrading, and cleaning of the skin above the palpated muscle bellies prior to electrode placement. After EMG placement, maximum voluntary isometric contractions (MVICs) were performed to determine the peak activation for each muscle following the SENIAM guidelines.

\section{Experimental procedures}

All participants performed back squats in 2 separate testing sessions, with/without knee sleeves, set 5-7 days apart. They executed 1RM and submaximal (80\% 1RM) back squats during both sessions. All subjects were provided with 7-mm Rehband knee sleeves (Rehband Stockholm AB, Stockholm, Sweden), fitted in accordance with the manufacturer's recommendations, depending on the upper calf circumference (XS: 31-33 cm, S: 33-35 cm, M: 35-37 cm, L: 37-40 cm, XL: 40-43 cm, XXL 43-46 cm). Counterbalancing for session/sleeve combinations was performed with a random number generator and pre-assigned to all participants. Counterbalancing was implemented to eliminate confounding variables/effects and limit the impact of any fatigue. The participants were directed to maintain their normal nutritional, sleep, and general activity habits. However, they were instructed to avoid any heavy squatting between sessions or high-volume body weight or light squatting of any variant (body weight squats, front squats, overhead squats, etc.).

The individuals were allowed 5 minutes for warming up and stretching of their choice. Next, they completed the National Strength and Conditioning Association's 1RM testing protocol [22]. They were given approximately 20 minutes to warm up to their $1 \mathrm{RM}$, beginning with a light resistance that allowed the participant to perform 5-10 repetitions with ease, followed by a 1-minute rest period. Then, the subjects lifted a warm-up load that allowed 3-5 repetitions by adding 30-40 lb (14-18 kg) or 10-20\%, followed by a 2-minute rest. Next, a conservative, near-maximal load was used, in which a participant could perform 2-3 repetitions after adding 30-40 lb (14-18 kg) or $10-20 \%$, followed by a $2-4-$ minute rest. Then, a $1 R M$ was attempted after another load increase of the same amount. If successful, the subject rested 2-4 minutes and another $30-40 \mathrm{lb}(14-18 \mathrm{~kg})$ or $10-20 \%$ increase was made for another attempt. If the participant failed, 2-4 minutes of rest were given; the mass was reduced by $15-20 \mathrm{lb}(7-9 \mathrm{~kg})$ or $5-10 \%$ and a $1 \mathrm{RM}$ was attempted again, until an official laboratory 1RM was found.

As it is impossible to shield one's knowledge of whether they are wearing knee sleeves, the following procedures were implemented to blind/restrict the participants' knowledge of the exact mass they were lifting. The subjects were required to face away from the bar as it was being loaded between the sets and anytime the participant was not actively lifting the bar. They were obliged to immediately approach the 
bar to perform the squat movement, without providing any time to stop and view the loaded barbell. All squats were to be performed to full depth (contact between posterior thigh and shank). The individuals were instructed to squat using a shoulder-width stance. 'Bouncing' out of the bottom of the squat was not permitted and was regulated by a command of 'up' upon thigh-calf contact. Spotters were available on each side of the participant during near-maximal and maximal lifts. A successful 1RM trial occurred when the athlete descended to full squat depth and successfully returned to a standing upright position.

After completion of 1RM testing, the participants were provided rest until they felt prepared for submaximal testing. Each individual performed 2 sets of 3 repetitions at $80 \%$ of their laboratory-tested 1RM for each condition (sleeves/no sleeves). Because not all exercisers perform back squats to full depth, the submaximal sets were completed under 2 squat depth conditions: full-depth and then parallel (thighs parallel to the floor). This procedure limited the impact of depth as a possible confounding variable. A 5-minute rest was enforced between the submaximal sets. In the second session (occurring 1 week later), all procedures from session 1 were repeated, but under the remaining condition of with/without knee sleeves.

\section{Data analyses}

All kinematic and GRF data were imported into and processed in commercial biomechanics software, Visual 3D Biomechanical Suite (v6.0, C-Motion, Germantown, USA). Three-dimensional marker trajectories and GRFs were filtered at a cut-off frequency of $5 \mathrm{~Hz}$ [2] with the use of a zero-lag fourth-order Butterworth low-pass filter. Power spectrum and residual analyses on shank marker trajectories indicated the optimal cut-off frequency should fall between 1 (too low) and $6 \mathrm{~Hz}$, respectively. The Davis method was utilized to determine hip joint centres [21, 23]. Knee and ankle joint centres were defined as the midpoints of the femoral epicondyles and malleoli, respectively. Direct kinematics served to compute joint angles. An X-Y-Z (extension-adduction-axial rotation) Cardan rotational sequence was used for angular kinematics computations. The conventions of kinematic and kinetic variables were determined with the right-hand rule. Joint moments were calculated with bottom-up inverse dynamics, normalized to the sum of body mass and mass lifted $(\mathrm{kg}){ }^{*}$ leg length $(\mathrm{m})$ and calculated as internal moments expressed in the distal segment $(\mathrm{Nm} / \mathrm{kgm})$.
GRFs were implemented to derive centre of gravity velocity using the relationship $\Sigma F=$ ma. First, the sum of body and barbell weights (measured as the sum of vertical force on the force platforms during the stationary period prior to beginning the squat) were subtracted from the vertical GRF. Next, the remaining force was divided by the sum of body and barbell masses, resulting in vertical acceleration. Vertical velocity was calculated as the first integral of acceleration (trapezoidal rule) beginning at full depth (when the participant was stationary, i.e. zero vertical velocity) and ending when the participant was standing upright.

The recorded muscle activations were imported to Visual 3D, high-pass filtered at $20 \mathrm{~Hz}$ [24], rectified, and low-pass filtered at $5 \mathrm{~Hz}$. Muscle activations during squats were normalized to the peak muscle activation record during the 7-second MVIC trials. Integrated EMG (iEMG; MVIC * seconds) was computed as the area under the normalized muscle activation waveforms during the descent and ascent phases. Descent was denoted as the instant centre of gravity began a downward trajectory (velocity exceeding $-0.01 \mathrm{~m} / \mathrm{s}$ ) and ended when the participant reached full squat depth. Full depth was denoted as the lowest point of the centre of gravity. In accordance with the requirements for back squat form (see above), this occurred when the posterior thigh contacted the posterior shank/leg. Ascent began at the instant the centre of gravity began to rise from full depth (velocity exceeding $0.01 \mathrm{~m} / \mathrm{s}$ ) and ended at the instant of peak centre of mass height. Variables of interest included: tri-planar knee angles, tri-planar knee moments, and iEMG during the descent (at $45^{\circ}$ knee flexion) and ascent phases (at $45^{\circ}$ knee flexion) and at full squat depth. Maximal mass lifted during 1RM squats and peak vertical velocity for both $1 \mathrm{RM}$ and submaximal loads were compared between conditions.

To determine the reliability of our motion capture methodology between sessions, root absolute differences, percent differences, and root mean square differences were computed for lower body segment lengths, proximal radii (e.g. knee joint radius), and distal radii (e.g. ankle joint radius) obtained from the static motion capture trials. Absolute difference and root mean square difference averages and $95 \%$ confidence intervals were $0.005 \pm 0.001 \mathrm{~m}$ and $0.008 \pm 0.001 \mathrm{~m}$, respectively.

\section{Statistical analyses}

Paired samples $t$-tests were utilized to compare mass lifted (kg), knee biomechanics, and muscle activations between sleeve and no sleeve conditions, and between 


\section{HUMAN MOVEMENT}

H.J. Bennett, A. Trypuc, K.A. Valenzuela, Z.A. Sievert, Knee sleeves do not affect knee mechanics

visits $\left(1^{\text {st }}\right.$ vs. $\left.2^{\text {nd }}\right)$ for $1 R M$ tests and body mass $(\mathrm{T}(14)$ for all tests). T-tests were also used to compare peak vertical velocity between conditions for both 1RM and submaximal loads. A two-way ANOVA with repeated measures on both factors was used to determine the effects of knee sleeves and squat depth on lower body biomechanics during submaximal squats $(\mathrm{F}(1,14)$ for interaction and main effects). In the presence of a significant interaction, post-hoc Tukey's honestly significant difference tests were applied to determine mean separations. The statistical significance of the results for all analyses was accepted at $p<0.05$. Effect sizes were reported for significant comparisons ( $t$-tests: Cohen's $d$; ANOVAs: partial eta-squared). The normality of the tested variables was analysed with Shapiro-Wilk tests, and no normality issues were found.

\section{Ethical approval}

The research related to human use has complied with all the relevant national regulations and institutional policies, has followed the tenets of the Declaration of Helsinki, and has been approved by the authors' institutional review board.

\section{Informed consent}

Informed consent has been obtained from all individuals included in this study.

\section{Results}

Ensemble figures are presented for 1RM (Figure 1), submaximal lift to full depth (Figure 2), and submaximal lift to parallel depth (Figure 3).

\section{Maximal squats}

No significant condition differences were found in $1 \mathrm{RM}$ or peak vertical velocity during maximal lifts or submaximal lifts (Table 1). No significant differences in 1RM $(p=0.76)$ or participant's mass $(p=0.95)$ were observed between the testing days.

No significant differences were found in knee joint angles at maximal depth between conditions (Table 2; $p>0.05)$. There were no significant differences in knee moments between conditions during descent or ascent, or at maximal depth (Table 3 ; all $p>0.05$ ).

Gluteus maximus iEMG during the ascent phase (full depth to standing) was significantly increased without knee sleeves compared with sleeves (Table 4; $t$-value: $2.11, p=0.04, d=0.74)$. No other differences were found in muscle activations during maximal squats (all $p>0.05$ ).

\section{Submaximal squats}

Knee flexion angle at depth was significantly greater during full depth compared with parallel squats for both conditions (Table 5; F $=119.05, p<0.01, \eta_{\mathrm{p}}{ }^{2}=$ $0.89)$. No other knee kinematics differences were found (all $p>0.05$ ). Knee external rotation moments during

Table 1. Mass lifted and peak vertical velocity comparisons between conditions: mean $\pm S D$

\begin{tabular}{lrrr} 
& No sleeves & Sleeves & $\begin{array}{c}\text { Test statistics } \\
(t, p, d)\end{array}$ \\
\cline { 2 - 4 } & & $121.4 \pm 29.5$ & $1.58,0.81,0.08$ \\
1RM (kg) & $119.1 \pm 28.2$ & $0.61 \pm 0.31$ & $0.34,0.74,0.15$ \\
Full_Submax VEL & $0.57 \pm 0.23$ & $0.67 \pm 0.19$ & $0.06,0.95,0.05$ \\
Par_Submax_VEL & $0.68 \pm 0.18$ & $0.64 \pm 0.26$ & $1.70,0.11,0.51$ \\
\hline
\end{tabular}

$S D$ - standard deviation, 1RM - 1-repetition maximum barbell mass lifted, VEL - peak vertical velocity during ascent phase, Full - full depth squats, Par - parallel squats

Table 2. Knee angles at full depth during 1RM squats: mean $\pm S D$

\begin{tabular}{lccc} 
& No sleeves & Sleeves & $\begin{array}{c}\text { Test statistics } \\
(t, p, d)\end{array}$ \\
\cline { 2 - 4 } Sag. & $-117.4 \pm 13.2$ & $-116.2 \pm 13.7$ & $0.67,0.51,0.09$ \\
Fron. & $12.5 \pm 6.1$ & $11.8 \pm 6.4$ & $0.52,0.61,0.11$ \\
Trans. & $15.9 \pm 12.4$ & $17.6 \pm 12.8$ & $0.77,0.46,0.13$ \\
\hline
\end{tabular}

Angle polarity follows right-hand rule.

$1 R M$ - 1-repetition maximum, SD - standard deviation, Sag. - sagittal plane, Fron. - frontal plane, Trans. - transverse plane 

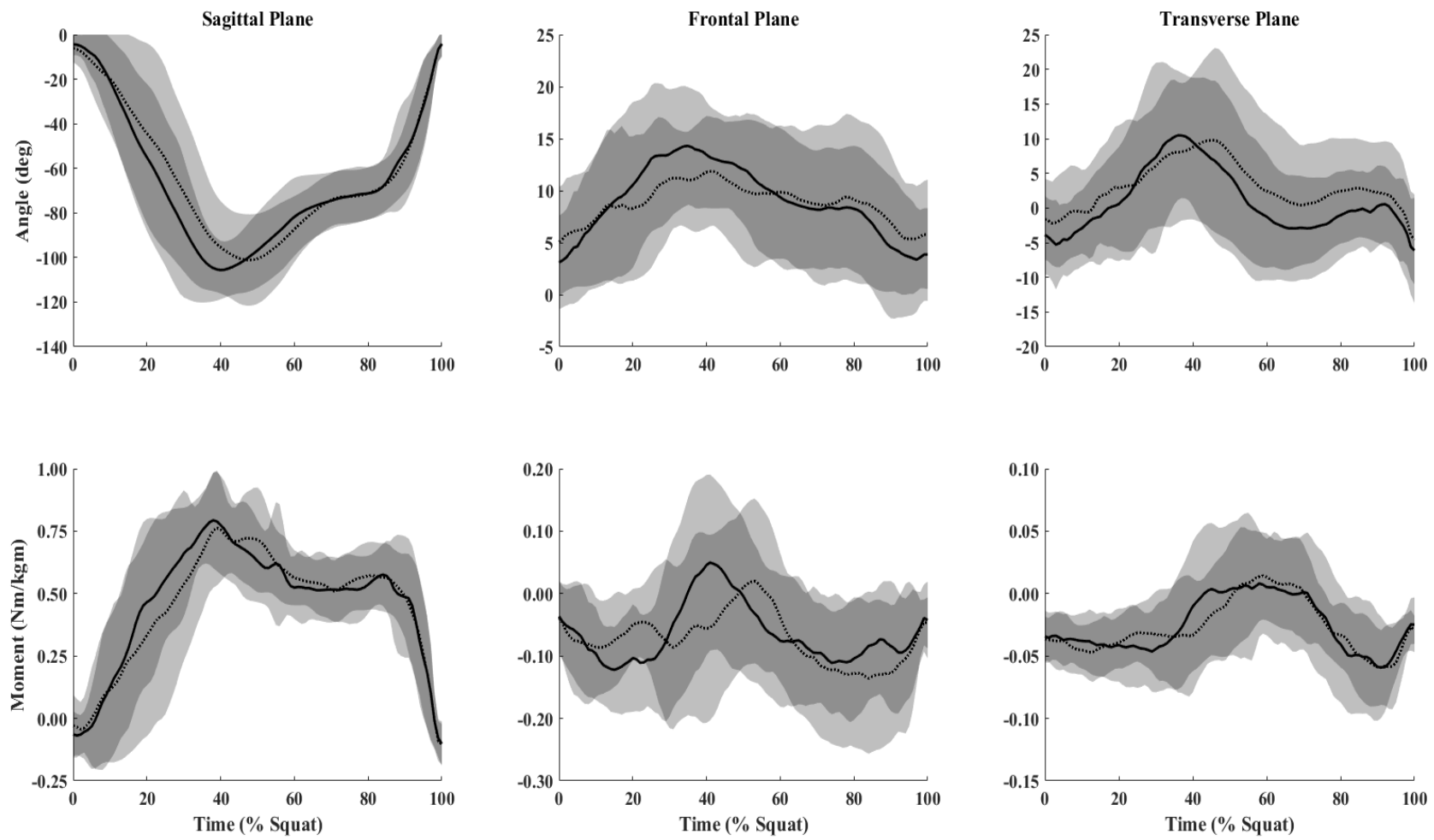

Figure 1. Ensemble knee biomechanics during 1RM squats with and without knee sleeves. Ensemble knee joint angles (top row) and moments (bottom row) are presented for conditions without knee sleeves (solid lines and dark shading) and with knee sleeves (dashed lines and light shading). Moments were normalized to the sum of body mass and barbell load lifted $(\mathrm{kg}) *$ leg length $(\mathrm{m})$
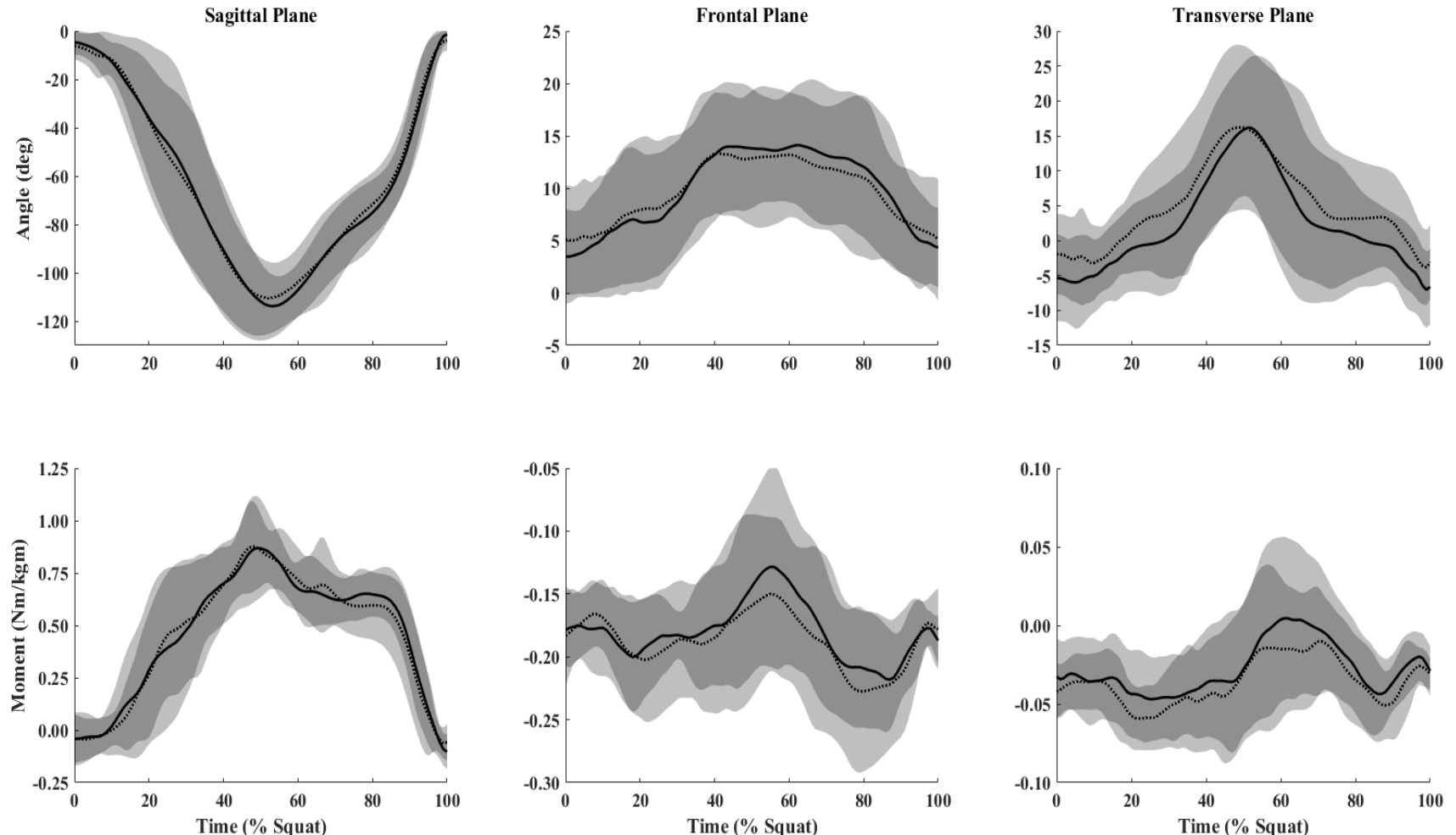

Figure 2. Ensemble knee biomechanics during submaximal squats to full depth with and without knee sleeves. Ensemble knee joint angles (top row) and moments (bottom row) are presented for conditions without knee sleeves (solid lines and dark shading) and with knee sleeves (dashed lines and light shading). Moments were normalized to the sum of body mass and barbell load lifted $(\mathrm{kg}){ }^{*}$ leg length (m) 


\section{HUMAN MOVEMENT}

H.J. Bennett, A. Trypuc, K.A. Valenzuela, Z.A. Sievert, Knee sleeves do not affect knee mechanics
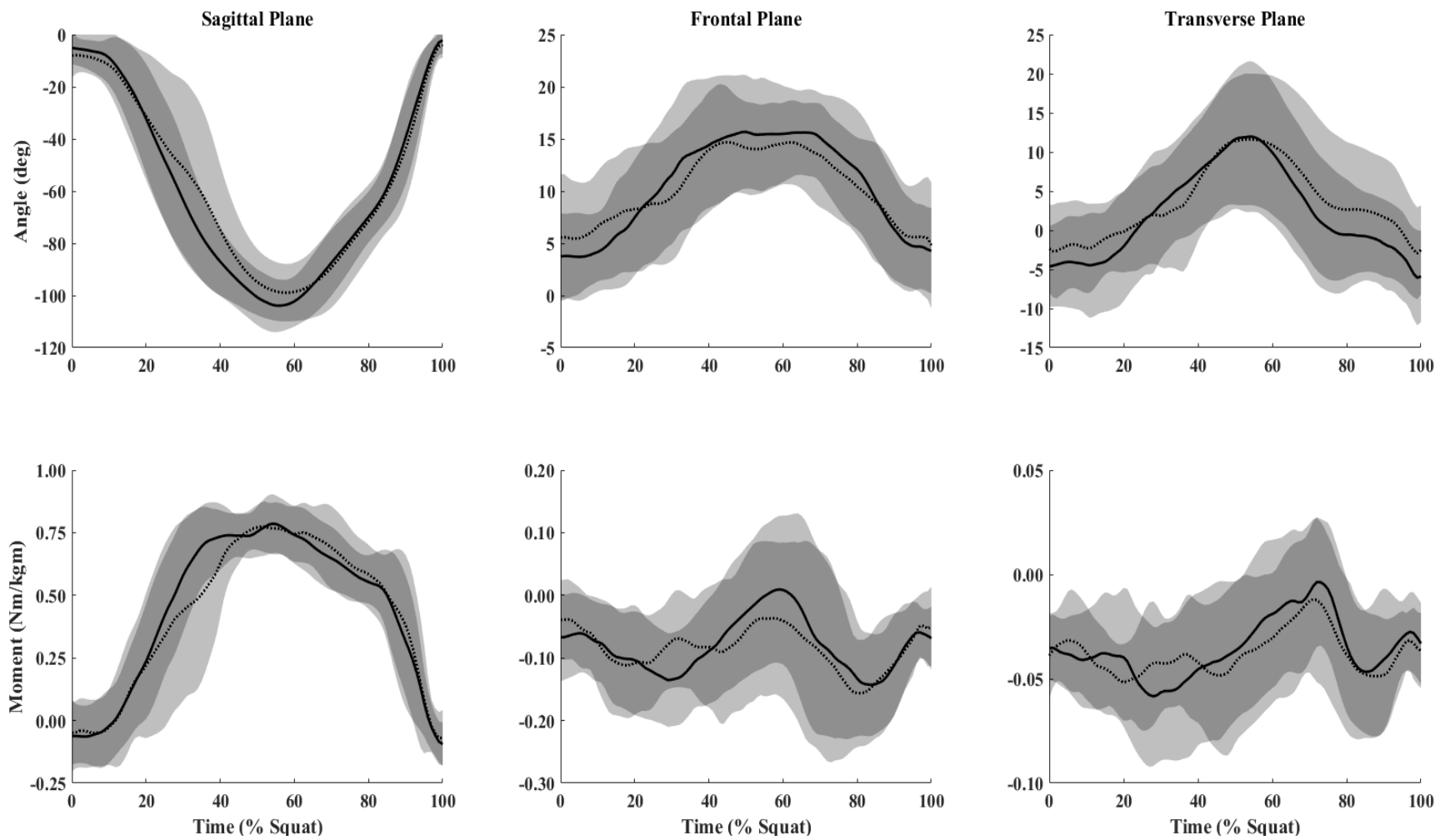

Figure 3. Ensemble knee biomechanics during submaximal squats to parallel with and without knee sleeves. Ensemble knee joint angles (top row) and moments (bottom row) are presented for conditions without knee sleeves (solid lines and dark shading) and with knee sleeves (dashed lines and light shading). Moments were normalized to the sum of body mass and barbell load lifted $(\mathrm{kg}) *$ leg length $(\mathrm{m})$

Table 3. Knee moments during 1RM squats: mean $\pm S D$

\begin{tabular}{ccc}
\hline No sleeves & Sleeves & Test statistics \\
& $(t, p, d)$
\end{tabular}

\begin{tabular}{|c|c|c|c|c|}
\hline \multirow{3}{*}{ 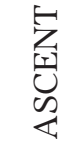 } & X & $0.57 \pm 0.15$ & $0.56 \pm 0.22$ & $0.21,0.83,0.08$ \\
\hline & $\mathrm{Y}$ & $-0.15 \pm 0.12$ & $-0.16 \pm 0.15$ & $0.79,0.44,0.19$ \\
\hline & $\mathrm{Z}$ & $-0.08 \pm 0.04$ & $-0.08 \pm 0.05$ & $0.08,0.94,0.00$ \\
\hline \multirow{3}{*}{ 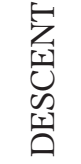 } & X & $0.57 \pm 0.10$ & $0.56 \pm 0.14$ & $0.62,0.55,0.00$ \\
\hline & $\mathrm{Y}$ & $-0.18 \pm 0.11$ & $-0.20 \pm 0.11$ & $1.09,0.33,0.33$ \\
\hline & $\mathrm{Z}$ & $-0.07 \pm 0.03$ & $-0.08 \pm 0.03$ & $0.01,0.99,0.00$ \\
\hline \multirow{3}{*}{ 舀 } & 18 & $1.17 \pm 0.33$ & $1.14 \pm 0.51$ & $0.13,0.90,0.16$ \\
\hline & $\mathrm{Y}$ & $0.08 \pm 0.20$ & $0.02 \pm 0.19$ & $1.94,0.07,0.33$ \\
\hline & Z & $-0.02 \pm 0.05$ & $-0.03 \pm 0.05$ & $0.82,0.43,0.22$ \\
\hline
\end{tabular}

1RM - 1-repetition maximum, $S D$ - standard deviation $\mathrm{X}, \mathrm{Y}$, and $\mathrm{Z}$ denote extension/flexion, ad-/ab-duction, and internal/external moments. Moments normalized to body mass + barbell mass $(\mathrm{kg}){ }^{*}$ leg length $(\mathrm{m})$. ASCENT and DESCENT denote variables measured at $45^{\circ}$ knee joint angles during ascent and descent phases of squat. Moment polarity follows right-hand rule.
Table 4. Muscle activation comparisons during 1RM squats: mean $\pm S D$

\begin{tabular}{|c|c|c|c|c|}
\hline & & No sleeves & Sleeves & $\begin{array}{l}\text { Test statistics } \\
\quad(t, p, d)\end{array}$ \\
\hline \multirow{5}{*}{ 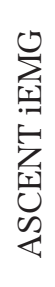 } & $\mathrm{RF}$ & $3.5 \pm 1.8$ & $2.9 \pm 1.8$ & $1.09,0.29,0.33$ \\
\hline & $\mathrm{VM}$ & $4.9 \pm 2.2$ & $4.7 \pm 3.4$ & $0.21,0.83,0.07$ \\
\hline & $\mathrm{BF}$ & $1.2 \pm 0.8$ & $1.1 \pm 0.7$ & $0.15,0.88,0.13$ \\
\hline & GMAX & $1.4 \pm 0.5$ & $1.0 \pm 0.5$ & $2.11,0.04,0.74$ \\
\hline & GMED & $1.1 \pm 0.5$ & $1.2 \pm 1.8$ & $0.32,0.75,0.08$ \\
\hline \multirow{5}{*}{ 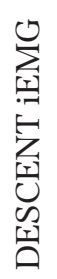 } & $\mathrm{RF}$ & $1.2 \pm 0.7$ & $1.1 \pm 0.8$ & $0.75,0.47,0.13$ \\
\hline & VM & $1.5 \pm 0.5$ & $1.4 \pm 0.6$ & $0.12,0.91,0.18$ \\
\hline & $\mathrm{BF}$ & $0.2 \pm 0.2$ & $0.2 \pm 0.2$ & $0.18,0.86,0.00$ \\
\hline & GMAX & $0.3 \pm 0.3$ & $0.2 \pm 0.1$ & $1.36,0.20,0.45$ \\
\hline & GMED & $0.5 \pm 0.5$ & $0.4 \pm 0.3$ & $0.55,0.59,0.24$ \\
\hline
\end{tabular}

1RM - 1-repetition maximum, $S D$ - standard deviation iEMG - integrated electromyography normalized to maximal activation during maximum voluntary isometric contractions trial

$\mathrm{RF}$ - rectus femoris GMAX - gluteus maximus $\mathrm{VM}$ - vastus medialis GMED - gluteus medius $\mathrm{BF}$ - biceps femoris 
Table 5. Knee angle comparisons at full depth during submaximal squats: mean $\pm S D$

\begin{tabular}{|c|c|c|c|c|c|c|c|}
\hline & \multicolumn{2}{|c|}{ No sleeves } & \multicolumn{2}{|c|}{ Sleeves } & \multirow{2}{*}{$\begin{array}{l}\text { Condition } \\
\left(\mathrm{F}, p, \eta_{\mathrm{p}}{ }^{2}\right)\end{array}$} & \multirow{2}{*}{$\begin{array}{c}\text { Depth } \\
\left(\mathrm{F}, p, \eta_{\mathrm{p}}^{2}\right)\end{array}$} & \multirow{2}{*}{$\begin{array}{c}\text { Interaction } \\
\left(\mathrm{F}, p, \eta_{\mathrm{p}}{ }^{2}\right)\end{array}$} \\
\hline & Full & Parallel & Full & Parallel & & & \\
\hline Sag. & $-120.0 \pm 11.7$ & $-106.3 \pm 9.9$ & $-118.3 \pm 13.3$ & $-107.0 \pm 11.3$ & $0.05,0.87,0.00$ & $119.05,<0.01,0.89$ & $0.13,0.69,0.01$ \\
\hline Fron. & $13.1 \pm 5.5$ & $15.6 \pm 4.8$ & $12.1 \pm 6.8$ & $14.6 \pm 4.9$ & $0.48,0.47,0.03$ & $4.33,0.09,0.24$ & $0.02,0.98,0.00$ \\
\hline Trans. & $18.4 \pm 11.4$ & $12.4 \pm 10.0$ & $19.3 \pm 12.1$ & $14.7 \pm 9.6$ & $0.27,0.57,0.02$ & $5.16,0.06,0.27$ & $0.07,0.81,0.00$ \\
\hline
\end{tabular}

Angle polarity follows right-hand rule.

$S D$ - standard deviation, Sag. - sagittal plane, Fron. - frontal plane, Trans. - transverse plane

Table 6. Knee moment comparisons during submaximal squats: mean $\pm S D$

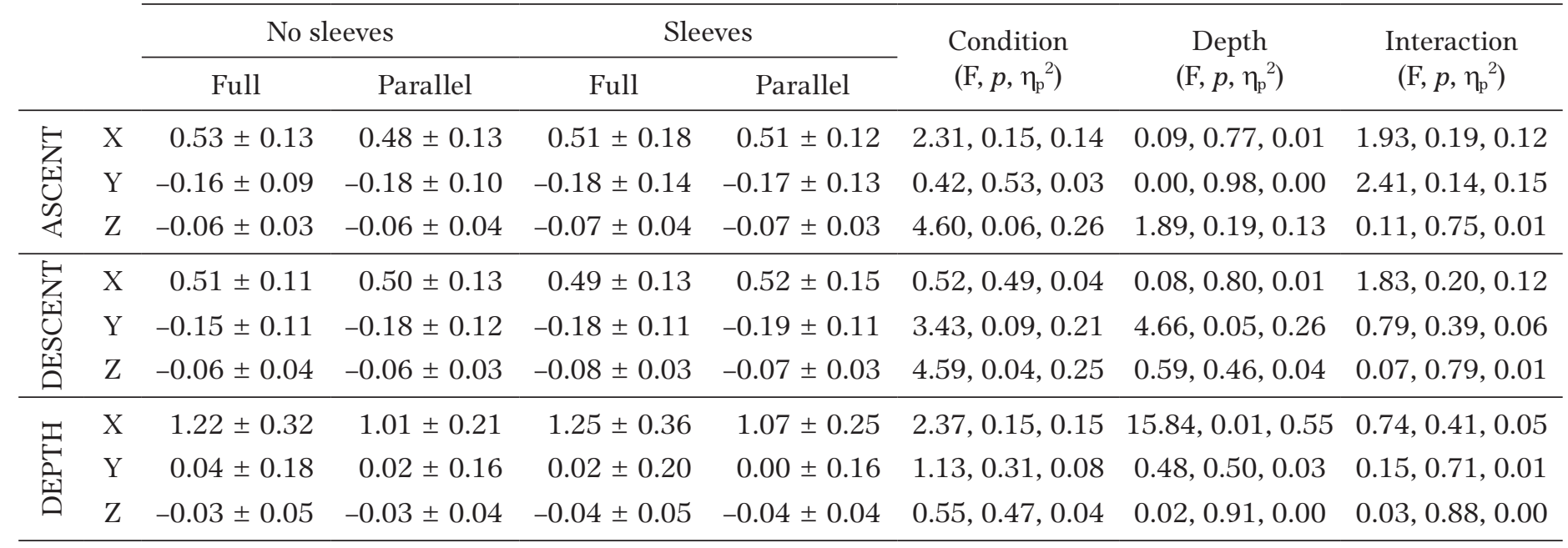

$\mathrm{X}, \mathrm{Y}$, and $\mathrm{Z}$ denote extension/flexion, ad-/ab-duction, and internal/external moments. Moments normalized to body mass + barbell mass $(\mathrm{kg}) *$ leg length $(\mathrm{m})$. ASCENT and DESCENT denote variables measured at $45^{\circ} \mathrm{knee} \mathrm{joint} \mathrm{angles} \mathrm{during} \mathrm{ascent}$ and descent phases of squat. Moment polarity follows right-hand rule.

$S D$ - standard deviation

Table 7. Muscle activation comparisons during submaximal squats: mean $\pm S D$

\begin{tabular}{|c|c|c|c|c|c|c|c|c|}
\hline & & \multicolumn{2}{|c|}{ No sleeves } & \multicolumn{2}{|c|}{ Sleeves } & \multirow{2}{*}{$\begin{array}{l}\text { Condition } \\
\left(\mathrm{F}, p, \eta_{\mathrm{p}}{ }^{2}\right)\end{array}$} & \multirow{2}{*}{$\begin{array}{c}\text { Depth } \\
\left(\mathrm{F}, p, \eta_{\mathrm{p}}{ }^{2}\right)\end{array}$} & \multirow{2}{*}{$\begin{array}{c}\text { Interaction } \\
\left(\mathrm{F}, p, \eta_{\mathrm{p}}{ }^{2}\right)\end{array}$} \\
\hline & & Full & Parallel & Full & Parallel & & & \\
\hline \multirow{5}{*}{ 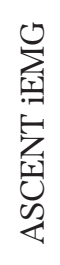 } & $\mathrm{RF}$ & $1.6 \pm 0.7$ & $1.4 \pm 0.4$ & $1.5 \pm 0.7$ & $1.5 \pm 0.7$ & $0.04,0.88,0.00$ & $0.32,0.54,0.02$ & $0.43,0.49,0.03$ \\
\hline & $\mathrm{VM}$ & $2.3 \pm 1.0$ & $2.0 \pm 0.7$ & $2.4 \pm 1.3$ & $2.5 \pm 1.5$ & $0.91,0.36,0.06$ & $0.10,0.74,0.01$ & $0.38,0.51,0.03$ \\
\hline & $\mathrm{BF}$ & $0.5 \pm 0.4$ & $0.5 \pm 0.4$ & $0.5 \pm 0.4$ & $0.5 \pm 0.3$ & $0.02,0.98,0.00$ & $0.05,0.85,0.00$ & $0.04,0.91,0.00$ \\
\hline & GMAX & $0.6 \pm 0.2$ & $0.5 \pm 0.2$ & $0.4 \pm 0.1$ & $0.4 \pm 0.1$ & $5.79,0.04,0.29$ & $0.19,0.63,0.01$ & $0.14,0.68,0.01$ \\
\hline & GMED & $0.5 \pm 0.3$ & $0.4 \pm 0.2$ & $0.4 \pm 0.3$ & $0.5 \pm 0.3$ & $0.09,0.75,0.01$ & $0.08,0.78,0.01$ & $0.16,0.66,0.01$ \\
\hline \multirow{5}{*}{ 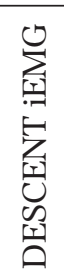 } & $\mathrm{RF}$ & $0.8 \pm 0.6$ & $1.0 \pm 0.5$ & $0.8 \pm 0.7$ & $1.0 \pm 0.9$ & $0.03,0.95,0.00$ & $1.82,0.24,0.12$ & $0.07,0.81,0.00$ \\
\hline & $\mathrm{VM}$ & $1.1 \pm 0.3$ & $1.3 \pm 0.5$ & $1.1 \pm 0.6$ & $1.4 \pm 0.8$ & $0.07,0.80,0.00$ & $5.16,0.06,0.27$ & $0.06,0.84,0.00$ \\
\hline & $\mathrm{BF}$ & $0.2 \pm 0.3$ & $0.3 \pm 0.3$ & $0.2 \pm 0.1$ & $0.2 \pm 0.2$ & $0.27,0.57,0.02$ & $0.45,0.48,0.03$ & $0.04,0.90,0.00$ \\
\hline & GMAX & $0.3 \pm 0.2$ & $0.3 \pm 0.2$ & $0.2 \pm 0.1$ & $0.3 \pm 0.2$ & $2.04,0.22,0.13$ & $0.40,0.50,0.03$ & $0.64,0.42,0.04$ \\
\hline & GMED & $0.4 \pm 0.2$ & $0.4 \pm 0.2$ & $0.4 \pm 0.3$ & $0.4 \pm 0.3$ & $0.36,0.52,0.03$ & $0.19,0.63,0.01$ & $0.03,0.97,0.00$ \\
\hline
\end{tabular}

$S D$ - standard deviation, iEMG - integrated electromyography normalized to maximal activation during maximum voluntary isometric contractions (MVIC) trial (MVIC * seconds)

$\mathrm{RF}$ - rectus femoris, VM - vastus medialis, BF - biceps femoris, GMAX - gluteus maximus, GMED - gluteus medius 
H.J. Bennett, A. Trypuc, K.A. Valenzuela, Z.A. Sievert, Knee sleeves do not affect knee mechanics

descent were larger with sleeves compared with no sleeves (Table 6; $\mathrm{F}=4.59, p=0.04, \eta_{\mathrm{p}}{ }^{2}=0.25$ ). Full depth squats produced increased knee extension moments at depth (Table 6; $\mathrm{F}=17.38, p=0.01, \eta_{\mathrm{p}}{ }^{2}=0.55$ ) compared with parallel squats. Similar to the 1RM lifts, gluteus maximus iEMG during ascent was larger in the no-sleeve compared with the sleeve condition (Table 7, F $=5.79, p=0.04, \eta_{\mathrm{p}}{ }^{2}=0.29$ ).

\section{Discussion}

The purpose of this study was to assess the effects of wearing neoprene knee sleeves on maximal mass lifted, knee biomechanics, and muscle activations during back squats. We hypothesized that using knee sleeves would increase 1RMs and bar speeds, reduce frontal and transverse plane loads, and reduce knee extensor activations. Our hypotheses were mostly rejected, as donning knee sleeves did not improve 1RM tests, increased knee external rotation moments during the descent phase of submaximal squats, and had no effect on knee muscle activations. However, integrated gluteus maximus activations were reduced when wearing knee sleeves.

Knee sleeves did not significantly improve $1 \mathrm{RMs}$ or bar speeds compared with not wearing sleeves. On a subject-specific basis, $1 \mathrm{RM}$ results were quite variable: 6 participants exhibited a greater $1 \mathrm{RM}$ with sleeves (range: $2.3-13.6 \mathrm{~kg}$ ), 3 participants presented a lower 1RM with sleeves (each $4.5 \mathrm{~kg}$ ), and 6 participants matched $1 \mathrm{RM}$ in both conditions. Given the effects of squat depth on difficulty in lifting similar loads [25], we also chose to investigate two different squat depths (full-depth: thigh-calf contact; and parallel: thighs parallel to ground). Similar to the previous report that analyzed knee sleeves when squatting to parallel [18], we found no improvement in any measured variable for knee sleeves during parallel or full depth squats.

Although little research exists on knee sleeves during weightlifting exercises, knee wraps are a well investigated knee support device. Various studies have found additional mass lifted with knee wraps to be anywhere from $10-13 \%$ to $20-25 \%$ while squatting to $90^{\circ}[5,10$, $12]$. Although $1 \mathrm{RMs}$ in our study were not performed to a depth of $90^{\circ}$, our comparisons of submaximal squats to both full-depth $\left(120^{\circ}\right)$ and parallel $\left(100^{\circ}\right)$ suggest no 'carry-over' effect would occur at smaller knee angles. Theoretically, knee sleeves should provide an elastic response that could increase mass lifted similar to knee wraps. However, this study revealed no increase in mass lifted. In addition, this study and the previous work by Sinclair et al. [18] found no increase in vertical velocity. The lack of differences observed here compared with previous studies' findings for knee wraps $[5,10,12]$ are likely due to differences in the elastic properties of these two materials. It is also important to note knee wraps are worn very tightly around the knee joint [5, 12], whereas knee sleeves worn in accordance with the manufacturer's specifications do not provide the same tight fit. However, regardless of fit, it is highly likely knee sleeves would never approach the advantages knee wraps provide. In this respect, coaches, athletes, and the general population should not consider knee sleeves as a device to improve weightlifting performance (i.e. 1RMs/ bar speeds in submaximal loads).

Previous works utilizing neoprene knee sleeves suggest knee sleeves are useful as knee support during less physical tasks $[13,15,17]$. The current literature indicates wearing knee sleeves reduces frontal plane loading during walking in persons with knee osteoarthritis [15] and limits the amount of anterior tibial translation during passive tibial translation tests in healthy athletes [17]. On the basis of previous reports, it would appear knee sleeves provide some frontal plane support for the knee joint during low-load tasks. Contrary to these studies, we found no changes in frontal plane knee biomechanics and observed an increase in transverse plane knee moments during squats. Thus, although previous benefits have been associated with wearing supportive knee sleeves in unloaded movements, these improvements do not translate to loaded back squats. Similar to the performance aspect, knee sleeves should not be considered as a device that provides frontal or transverse plane support. Persons that are seeking/need additional frontal plane support should consider other devices, such as knee braces.

The lack of activation differences in knee extensors and flexors during 1RM or submaximal squats was surprising. If knee sleeves were to provide a discernible mechanical advantage, they should have released stored elastic energy from the descent phase into the ascent phase, assisting knee muscle effort during $1 \mathrm{RMs}$ and increasing the mass lifted, while reducing knee muscle activations during submaximal squats. Neither of these effects was observed. Although no EMG was assessed, the previous study by Sinclair et al. [18] found no differences in predicted muscle forces with knee sleeves compared with the natural condition. On the basis of our recorded muscle activations and the muscle force predictions in the previous study [18], knee sleeves provide no advantage for muscles surrounding the knee joint. 
Interestingly, gluteus maximus iEMG during the ascent phase of both 1RM and submaximal squats was significantly reduced when using sleeves, which is the opposite effect to that recorded with knee wraps [9]. Given the large involvement of the hip joint/musculature in deep squats $[2,19,20]$, reducing gluteus muscle activation when squatting with the same resistance/load could indicate a mechanical advantage of knee sleeves. However, coupled with the lack of increased 1RM mass lifted/bar speeds and the lack of alterations in quadricep or hamstring muscle activations, knee sleeves appear to provide no significant advantage to the neuromuscular system. Contrary to our findings, wearing knee wraps at 90\% 1RM squats resulted in lower activation of the vastus lateralis, but no difference in integrated gluteus maximus activity compared with no knee wraps [5]. Therefore, despite similarities in usage/application, knee sleeves do not appear to provide the same effects as knee wraps.

There are limitations to acknowledge with this study. First, our participant pool included only persons with a history of back squatting ( $\geq 1$ year) and familiarity with using knee sleeves. It is possible that the effects of knee sleeves will be altered when implemented in a less familiarized weightlifter. Second, we did not perform mechanical testing on the knee sleeves; thus, the actual recoil and stored elastic energies of this material are currently unknown to the authors. Lastly, this study only included a shoulder-width squat stance; therefore, the results are only applicable to shoulderwidth stance.

\section{Conclusions}

In general, this study found neoprene knee sleeves did not aid in maximal performance or increased frontal plane knee joint stability during back squats. Mass lifted did not change with the application of knee sleeves. In addition, neither frontal and transverse plane knee biomechanics nor the surrounding knee muscles were significantly impacted. However, gluteus maximus activation decreased with the application of the knee sleeves during both 1RM and submaximal squats.

\section{Acknowledgements}

The authors would like to acknowledge Rehband for donating the knee sleeves for biomechanical analyses.

\section{Disclosure statement}

No author has any financial interest or received any financial benefit from this research.

\section{Conflict of interest}

The authors state no conflict of interest.

\section{References}

1. Escamilla RF. Knee biomechanics of the dynamic squat exercise. Med Sci Sports Exerc. 2001;33(1):127-141; doi: 10.1097/00005768-200101000-00020.

2. Escamilla RF, Fleisig GS, Lowry TM, Barrentine SW, Andrews JR. A three-dimensional biomechanical analysis of the squat during varying stance widths. Med Sci Sports Exerc. 2001;33(6):984-998; doi: 10.1097/0000 5768-200106000-00019.

3. Flanagan S, Salem GJ, Wang M-Y, Sanker SE, Greendale GA. Squatting exercises in older adults: kinematic and kinetic comparisons. Med Sci Sports Exerc. 2003;35(4):635-643; doi: 10.1249/01.MSS.00000583 64.47973.06.

4. Schoenfeld BJ. Squatting kinematics and kinetics and their application to exercise performance. J Strength Cond Res. 2010;24(12):3497-3506; doi: 10.1519/JSC. 0b013e3181bac2d7.

5. Gomes WA, Brown LE, Soares EG, da Silva JJ, de O Silva FHD, Serpa ÉP, et al. Kinematic and SEMG analysis of the back squat at different intensities with and without knee wraps. J Strength Cond Res. 2015;29(9): 2482-2487. doi: 10.1519/JSC.0000000000000922.

6. Flanagan SP, Salem GJ. Lower extremity joint kinetic responses to external resistance variations. J Appl Biomech. 2008;24(1):58-68; doi: 10.1123/jab.24.1.58.

7. Fry AC, Smith JC, Schilling BK. Effect of knee position on hip and knee torques during the barbell squat. J Strength Cond Res. 2003;17(4):629-633; doi: 10.1519/ 1533-4287(2003)017<0629:eokpoh>2.0.co;2.

8. Lorenzetti S, Gülay T, Stoop M, List R, Gerber H, Schellenberg F, et al. Comparison of the angles and corresponding moments in the knee and hip during restricted and unrestricted squats. J Strength Cond Res. 2012;26(10):2829-2836; doi: 10.1519/JSC.0b013e318 267918b.

9. Lake JP, Carden PJC, Shorter KA. Wearing knee wraps affects mechanical output and performance characteristics of back squat exercise. J Strength Cond Res. 2012;26(10):2844-2849; doi: 10.1519/JSC.0b013e318 2429840.

10. Marchetti PH, Pereira Matos VJ, Soares EG, da Silva JJ, Serpa ÉP, Corrêa DA, et al. Can the technique of knee wrap placement affect the maximal isometric force during back squat exercise? Int J Sports Sci. 2015;5(1):16-18; doi: 10.5923/j.sports.20150501.03.

11. Totten L. Knee wraps. Strength Cond J. 1990;12(5): 36-39.

12. Harman E, Frykman P. Bridging the gap - research: the effects of knee wraps on weightlifting performance and injury. Strength Cond J. 1990;12(5):30-35.

13. Bryk FF, de Jesus JF, Fukuda TY, Moreira EG, Marcondes FB, dos Santos MG. Immediate effect of the elastic knee sleeve use on individuals with osteoarthritis. 


\section{HUMAN MOVEMENT}

H.J. Bennett, A. Trypuc, K.A. Valenzuela, Z.A. Sievert, Knee sleeves do not affect knee mechanics

Rev Bras Reumatol. 2011;51(5):440-446; doi: 10.1590/ S0482-50042011000500004.

14. Mazzuca SA, Page MC, Meldrum RD, Brandt KD, Petty-Saphon S. Pilot study of the effects of a heat-retaining knee sleeve on joint pain, stiffness, and function in patients with knee osteoarthritis. Arthritis Rheum. 2004;51(5):716-721; doi: 10.1002/art.20683.

15. Schween R, Gehrig D, Gollhofer A. Immediate effects of an elastic knee sleeve on frontal plane gait biomechanics inkneeosteoarthritis.PLoSOne.2015;10(1):e0115782; doi: 10.1371/journal.pone.0115782.

16. Van Tiggelen D, Coorevits P, Witvrouw E. The use of a neoprene knee sleeve to compensate the deficit in knee joint position sense caused by muscle fatigue. Scand J Med Sci Sports. 2008;18(1):62-66; doi: 10.1111/ j.1600-0838.2007.00649.x.

17. Csapo R, Hosp S, Folie R, Eberle R, Hasler M, Nachbauer W. Elastic knee sleeves limit anterior tibial translation in healthy females. J Sports Sci Med. 2016;15(1): 206-207.

18. Sinclair J, Mann J, Weston G, Poulsen N, Edmundson CJ, Bentley I, et al. Acute effects of knee wraps/ sleeve on kinetics, kinematics and muscle forces during the barbell back squat. Sport Sci Health. 2020;16:227237; doi: 10.1007/s11332-019-00595-5.

19. Caterisano A, Moss RF, Pellinger TK, Woodruff K, Lewis VC, Booth W, et al. The effect of back squat depth on the EMG activity of 4 superficial hip and thigh muscles. J Strength Cond Res. 2002;16(3):428-432; doi: 10.1519/1533-4287(2002)016<0428:TEOBSD>2.0.CO;2.

20. Contreras B, Vigotsky AD, Schoenfeld BJ, Beardsley C, Cronin J. A comparison of gluteus maximus, biceps femoris, and vastus lateralis electromyography amplitude in the parallel, full, and front squat variations in resistance-trained females. J Appl Biomech. 2016;32(1): 16-22; doi: 10.1123/jab.2015-0113.

21. Bennett HJ, Fleenor K, Weinhandl JT. A normative database of hip and knee joint biomechanics during dynamic tasks using anatomical regression prediction methods. J Biomech. 2018;81:122-131; doi: 10.1016/j. jbiomech.2018.10.003.

22. Haff GG, Triplett NT. Essentials of strength training and conditioning, $3^{\text {rd }}$ ed. Champaign: Human Kinetics; 2016.

23. Davis RB, Õunpuu S, Tyburski D, Gage JR. A gait analysis data collection and reduction technique. Hum Mov Sci. 1991;10(5):575-587; doi: 10.1016/0167-9457(91) 90046-Z.

24. De Luca CJ, Gilmore LD, Kuznetsov M, Roy SH. Filtering the surface EMG signal: movement artifact and baseline noise contamination. J Biomech. 2010;43(8):15731579; doi: 10.1016/j.jbiomech.2010.01.027.

25. Wretenberg P, Feng Y, Arborelius UP. High- and low-bar squatting techniques during weight-training. Med Sci Sports Exerc. 1996;28(2):218-224; doi: 10.1097/0000 5768-199602000-00010.

\section{APPENDIX}

Table 1. Raw knee joint moments during $1 \mathrm{RM}$ and submaximal squats: mean $\pm S D$

\begin{tabular}{|c|c|c|c|c|c|c|c|}
\hline & & \multicolumn{2}{|c|}{ No sleeves } & \multicolumn{2}{|c|}{ Submaximal full } & \multicolumn{2}{|c|}{ Submaximal parallel } \\
\hline & & No sleeves & Sleeves & No sleeves & Sleeves & No sleeves & Sleeves \\
\hline \multirow{3}{*}{ 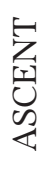 } & $\mathrm{X}$ & $111.32 \pm 35.63$ & $115.55 \pm 41.81$ & $80.83 \pm 30.22$ & $85.04 \pm 32.17$ & $77.69 \pm 28.46$ & $85.46 \pm 28.36$ \\
\hline & $\mathrm{Y}$ & $-31.44 \pm 19.64$ & $-34.51 \pm 24.78$ & $-25.21 \pm 16.30$ & $-28.87 \pm 20.84$ & $-31.94 \pm 15.20$ & $-28.31 \pm 20.21$ \\
\hline & $\mathrm{Z}$ & $-14.98 \pm 8.08$ & $-16.10 \pm 10.69$ & $-9.59 \pm 5.03$ & $-12.78 \pm 6.50$ & $-11.36 \pm 6.53$ & $-12.26 \pm 4.80$ \\
\hline \multirow{3}{*}{ 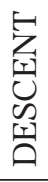 } & $\mathrm{X}$ & $104.92 \pm 33.26$ & $108.99 \pm 40.39$ & $77.88 \pm 26.30$ & $75.75 \pm 24.47$ & $81.36 \pm 21.86$ & $80.60 \pm 29.14$ \\
\hline & $\mathrm{Y}$ & $-37.46 \pm 22.54$ & $-42.90 \pm 21.56$ & $-28.25 \pm 21.13$ & $-32.41 \pm 20.76$ & $-31.27 \pm 20.85$ & $-32.00 \pm 20.69$ \\
\hline & $\mathrm{Z}$ & $-14.07 \pm 6.55$ & $-15.16 \pm 8.01$ & $-8.88 \pm 5.71$ & $-11.53 \pm 6.66$ & $-9.04 \pm 5.48$ & $-11.73 \pm 6.05$ \\
\hline \multirow{3}{*}{ 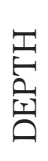 } & $\mathrm{X}$ & $224.66 \pm 81.80$ & $240.07 \pm 107.99$ & $193.06 \pm 88.91$ & $206.87 \pm 92.59$ & $158.78 \pm 46.37$ & $173.60 \pm 56.11$ \\
\hline & $\mathrm{Y}$ & $13.47 \pm 39.41$ & $1.27 \pm 39.81$ & $10.33 \pm 30.22$ & $4.39 \pm 33.72$ & $6.16 \pm 25.09$ & $2.87 \pm 26.72$ \\
\hline & $\mathrm{Z}$ & $-3.08 \pm 11.35$ & $-5.57 \pm 10.59$ & $-3.81 \pm 9.00$ & $-5.12 \pm 8.95$ & $-3.60 \pm 6.66$ & $-5.25 \pm 6.32$ \\
\hline
\end{tabular}

Submaximal squats performed at 80\% 1RM. Full and parallel refer to the depth of squats. X, Y, and Z denote extension/ flexion, ad-/ab-duction, and internal/external moments. ASCENT and DESCENT denote variables measured at $45^{\circ} \mathrm{knee}$ joint angles during ascent and descent phases of squat. Moment polarity follows right-hand rule.

$1 \mathrm{RM}$ - 1-repetition maximum, $S D$ - standard deviation 